\title{
Impact of Vehicular Integration Effects on the Performance of DSRC Communications
}

\author{
Jérôme Härri*, Hugues Tchouankem ${ }^{\S}$, Oliver Klemp**, Oleksandr Demchenko\| \\ ${ }^{*}$ Mobile Communications Department, EURECOM ${ }^{\dagger}$, France \\ $\S$ Institute of Communications Technology, Leibniz Universität Hannover, Germany \\ **BMW Group Research \& Technology, München, Germany \\ "Bosch SoftTec GmbH, Hildesheim, Germany
}

\begin{abstract}
Future safety-related vehicular applications require reliable information exchange provided by Dedicated Short Range Communication (DSRC). As the wireless vehicular environment is very challenging, it is crucial to test and evaluate the performance of DSRC communications in real conditions through experimental tests. Yet, DSRC communications have been evaluated independently from vehicles, and precise knowledge related to vehicular integration effects (i.e. cable/connector loss, antenna placement, vehicle geometry, etc) on DSRC communications still lacks.

In this paper, we measure aspects of vehicular integration influencing DSRC communication in field experimental tests. Considering a global link budget, we illustrate the non-negligible impacts of cable, connectors, hardware, or roof types and antenna design on the communication range. We also measure their influence on the wireless channel and show that a reasonable distance between vehicles and radio obstacles, or metallic sidereflector improve communication range. Applying these results to a Road Hazard Warning application, we illustrate that, while vehicular integration affects its performance, wireless channel effects, in particular side reflectors, partially compensate them.
\end{abstract}

\section{INTRODUCTION}

DSRC is the standard for dedicated inter-vehicular communications, and is a promising technology to improve communications for Intelligent Transportation Systems (ITS).

ITS traffic safety applications are the current focus of the applicability of DSRC technologies to improve safety on the road and reduce human death toll. However, DSRC prove to be subject to unreliable communication and to fluctuating transmission ranges, especially when the vehicular wireless channel becomes congested. As DSRC is responsible for the transmission of safety-of-life information, evaluations of the capabilities of DSRC in real conditions should be conducted, and advanced communication techniques should be developed to mitigate stringent wireless channel effects and to provide key performance indicators to traffic safety applications. With the recent bandwidth allocation of $30 \mathrm{MHz}$ at $5.9 \mathrm{GHz}$ for ITS in Europe, Vehicle-to-Vehicle (V2V) communication systems are close to market introduction.

Over the past years, DSRC went over deep investigations about its communication capabilities in various environments, first through simulations, but more recently in small scale

†EURECOM acknowledges the support of its industrial members: Cisco, BMW Group, Monaco Telecom, Orange, SAP, SFR, STMicroelectronics, Swisscom, Symantec. field trials, experimental tests and channel measurement campaigns [1-5]. Large amounts of expertise have been gathered, in particular on DSRC optimal operational conditions, and several solutions have been proposed to maintain such conditions regardless of the communications environment.

Yet, these studies have been conducted considering the DSRC technology independently and apart from a more global communication chain of the integration of the DSRC technology into vehicles. A link budget reflecting only the wireless side is not representative of the real capabilities of DSRC, as it would not include for instance, the influence of antenna design or cable and connector losses. Also, channel conditions have so far been assumed to be penalizing DSRC by strong fading from static and mobile radio obstacles [4, 5]. Indeed, communicating at $5.9 \mathrm{GHz}$ is challenging for non-line-of-sight (NLOS) transmissions, as waves are blocked and reflected by almost any obstacle. Yet, such reflection may also be beneficial, notably to provide communication around obstacles. The vehicular environment contains plenty of potential reflectors, which influence on the communication range should be investigated, in particular in conjunction with non-uniform antenna gains. The directional behavior of the antenna defines the reliability of safety-related communications between moving vehicles and as such the end-to-end arrival success of exchanged message types. Impairments in the directional behavior of automotive antenna equipment caused by vehicular mounting effects may degrade the link performance between transmitting $(\mathrm{TX})$ and receiving $(\mathrm{RX})$ vehicles as described in [6].

In this paper, we first formulate a link budget chain, containing all factors influencing integrated DSRC in vehicles. We classify them in different categories to be able to evaluate their influence independently. We then measure the link budget of each of them in a controlled laboratory environment, and extract typical values that may later be used by the community. We experimentally test the link budget of the antenna category in a detailed field test and identify the significant influence of the antenna design and integration on vehicles on the total link budget. Depending on the antenna and location, communication range may be significantly altered on various directions. Finally, we conduct a small scale field operational test in a specialized test track to evaluate the influences of integration factors on vehicular communication in term of 
Received Power levels and Packet Delivery Ratio, first without reflectors and then also including them. Consider the ITS Road Hazard Warning (RHW) application, we show that such factors have the capabilities to make it fail to detect danger with enough distance. Yet, our field test also showed that reflectors are providing a positive influence to the application success, and potentially counterbalancing the influence of integration factors. Considering that the integration factors are specific to each vehicle and technology, our conclusion is that the evaluation of the influence of DSRC requires a careful analysis of DSRC link budget, and this on a caseby-case basis. Also, we demonstrated that side-road metal reflectors are able to improve the link budget and as such the communication range by a factor of about two, bringing experimental communication conditions a bit less dramatic than previous studies.

The rest of this paper is organized as follows. In Section II, we classified the factors influencing the total link power budget of integrated DSRC. In Section III, we describe our test scenario and the specifications of the hardware under test. Section IV presents laboratory measurements. We provide results of a detailed antenna measurement field trials in Section $\mathrm{V}$, before describing the influence of the integration factor in a field operational test in Section VI. Section VII puts our results into perspective of ITS applications, and Section VIII concludes our work.

\section{Link Power Budget AnAlysis}

This section provides information on evaluating the complete link power budget chain, containing all factors influencing the quality of DSRC. Typically, a link power budget is defined as the sum of all gains and losses in the end to end communication link between two stations including antenna gain, cable and connector losses and the path between stations. A noisy transceiver or huge cable losses may affected considerably the performance of the network reflected by the limitation of the theoretically achievable communication range even in line-of-sight (LOS) situations. Furthermore, a credible simulation-based evaluation of DSRC requires a complete understanding of the link-budget. However, a correct calculation of the end-to-end link power budget is a complicated task, as it depends on a range of physical factors which severely affect the performance of DSRC by reducing the Signal to interference plus noise ratio (SINR).

Figure 1 depicts a overview of these factors which can be classified in four link building blocks including transceiver architecture, vehicle construction, antenna structure and channel condition.

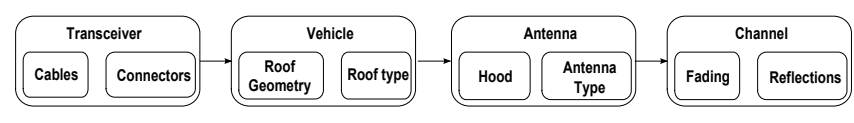

Figure 1: Block diagram of system factors.

The transceiver architecture factor, which depends on the mounting methodology of the radio module, may caused additional cable and connector losses. While the radio link quality may inevitably suffer from losses when the radio module is installed inside the vehicle (e.g. in rear trunk), an integrated radio module mounted directly on the vehicle's roof may significantly reduce additional cable and connector losses. The second factor, the vehicle structure, is described by factors related to the roof geometry and type of the vehicle, since it is show in [7] and [8] that the physical dimensions of vehicles and their roof type have an influence on the received signal power. The third factor is caused by antenna design methodology and describes a characteristic gain that is dependent on the geometry, type and hood material of the antenna as well as the placement of the antenna at the car's roof as discussed in section V. The last factor, channel characteristics, which is the most critical factor influencing the end-to-end link-budget, includes by the propagation of electromagnetic signals and the vehicular environment (urban, rural or highway). It first accounts for the attenuation of the transmitted signal caused by the decrease of the received signal power, which is proportional to the inverse of the distance between stations. It also accounts for channel gains such as fading, which is caused by constructive/destructive interferences amplifying/attenuating the received signal power in multipath scenarios, especially in urban environments.

In this paper, we will evaluate the influence of the transceiver architecture, vehicle roof type and channel characteristics through experimental tests conducted in laboratory and also on a controlled test track.

\section{Configuration of Test Environment}

\section{A. Hardware}

With respect to limitations in reproducibility and traffic synchronization, we opted against a setup with two vehicles independently moving against each other. Our solution is a discretization of the transmitter (TX) position, with the receiver (RX) driving in the test scenario. A BMW 5 Series was configured as receiver. A tripod with $35 \times 35 \mathrm{~cm}$ metal plate at a height of $1.45 \mathrm{~m}$ was used at TX position representing the approximate height of conventional vehicles acts as transmitter. The antenna element is represented by a $\lambda / 4$-monopole centered on a cylindrical ground plane with a diameter of $1 \mathrm{~m}$ and rounded edges to reduce edge diffraction. Both transmitter and receiver were equipped with a radio communication module that operates in the $5.9 \mathrm{GHz}$ frequency band and implements the IEEE 802.11p wireless standard, which introduces the newly defined Outside the Context of a Basic Service Set (OCB) mode [9]. The receiving vehicle was also equipped with a Differential Global Positioning System (DGPS) receiver providing a high accuracy position, in order to $\log$ the vehicle positions used to computed the distance to the TX-Station. The transmitting station was configured to send periodic position messages with a generation rate of $500 \mathrm{~Hz}$. A script was implemented at the receiver that were then used to record for each successfully received frame, the received signal power, the receiver position as well as the sequence number used to compute the packet delivery ratio (PDR). An additional script was implemented to adapt 
Table I: Hardware used and measurement system parameters

\begin{tabular}{l|l}
\hline Roof Type & metal, panorama-glas \\
\hline RX Antenna Type & Kathrein \\
\hline TX Frequency & $5.9 \mathrm{GHz}$ \\
\hline Bandwidth & $10 \mathrm{MHz}$ \\
\hline TX Power & $23 \mathrm{dBm}$ \\
\hline TX Antenna & $\lambda / 4-$ monopole \\
\hline Cable Loss $L_{c b}$ & $2.4 \mathrm{~dB}(0.6 \mathrm{~dB} / \mathrm{m})$ \\
\hline Splitter Fading $L_{s p l}$ & $2.1 \mathrm{~dB}$ \\
\hline Connector Fading $L_{c o n}$ & $0.5 \mathrm{~dB}$ \\
\hline RX Sensitivity & $-85 \mathrm{dBm}$ \\
\hline
\end{tabular}

the parameter settings and control the measurements during the field operating test. A summary of the most important hardware used and system parameters is presented in Table I.

\section{B. Test Scenario Description}

We evaluate the impact of the integration and channel effects in three methodologies as summarized in Table II:

- Lab Test - Transmitter and receiver radio modules are connected by a coaxial cable, including adjustable attenuators and a spectrum analyzer.

- Antenna Field Test - The test vehicle is static but turns over itself around a test antenna.

- Field Test - The TX-Station is located at a fixed position, while the receiving vehicle moves with a speed of approximately $45-50 \mathrm{~km} / \mathrm{h}$

The field operational test was classified in three scenarios as depicted in Fig. 3 and was carried out on the BMW test track in the Munich area in Autumn 2011. Additionally, all experiments were performed in a strong LOS environment in order to avoid or minimize the effect of buildings and other moving traffic.

- Scenario 1 - We first consider a open field, where the vehicle is approaching the TX-Station.

- Scenario 2 - The TX-Station is hidden behind a large truck, first $1 \mathrm{~m}$ behind and second $10 \mathrm{~m}$ behind.

- Scenario 3 - Similar to Scenario 2, but with a side reflector between the truck and the Tx-Station.

Table II: Tested metrics and methodology

\begin{tabular}{l|l|l}
\hline Methodology & Tested Values & KPI \\
\hline \hline Lab Test & cable, connectors & dB loss \\
\hline Antenna Field Test & $\begin{array}{l}\text { Roof geometry and type, } \\
\text { Antenna hood and gain }\end{array}$ & Antenna radiation \\
\hline Field Test & $\begin{array}{l}\text { Global Link Budget, } \\
\text { Channel Fading and } \\
\text { Reflection }\end{array}$ & Communication Range \\
\hline
\end{tabular}

\section{CABle And Connector Loss}

In the objective to provide realistic values of each component of the total link budget previously described, we built up a lab test depicted in Fig. 2. The RX side has two branches: a wireless chipset and a spectrum analyzer. The reason for this differentiation is to also test the influence of the chipset in the link-budget. Accordingly, we represent the hardware link budget as follows:

$$
\begin{aligned}
P_{\text {spec }}^{R X} & =P^{T X}-L_{c b}-L_{a t t}-L_{s p l}-L_{c o n} \\
P_{\text {wireless }}^{R X} & =P^{T X}-L_{c b}-L_{a t t}-L_{s p l}-L_{c b}
\end{aligned}
$$

, where $\mathrm{L}_{a t t}=40 \mathrm{~dB}$, modulation coding is QPSK, a 300 Bytes packet size, and all other parameters could be found in Table I. Please note that fading from cables, connectors and splitter have been taken from hardware (HW) specifications. We illustrate the output from the spectrum analyzer in Table III. We can first observe that the measured losses from HW do not correspond to the total link budget, but varies as function of the TX parameters. Yet, on average, the values do not fall too far apart, but still reach a $5.5 \mathrm{~dB}$ loss. Second, we can also observe that there is a difference between the received power (RX-Power) between the spectrum analyzer and wireless chipset, which cannot only be explained by the extra cable loss. Such loss comes from the wireless chipset and should also be considered in the link budget, as it may bring up to $2.4 \mathrm{~dB}$ extra loss in the overall link budget.

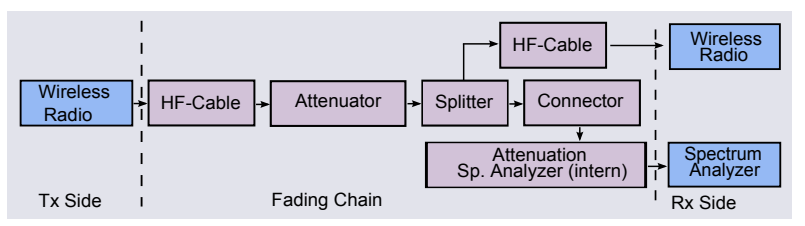

Figure 2: Illustration of the laboratory test, containing a wireless receiver and a spectrum analyzer

From our lab test, we can conclude that cable and connector losses must be considered in the link budget as they bring a significant impact on the link budget. More interesting, we should also consider an extra HW loss from the wireless chipset around $2 \mathrm{~dB}$. Whereas bringing the chipset closer to the antenna helps reducing losses from cables and connectors, such HW loss must be considered in the link budget.

Table III: HW impact in lab test, QPSK modulation, 300 Bytes packet size, $40 \mathrm{~dB}$ attenuation

\begin{tabular}{c|c|c|c|c|c|c}
\hline & \multicolumn{2}{|c|}{ Spectrum Analyzer } & \multicolumn{2}{|c|}{ Wireless Radio } & Chipset \\
\cline { 2 - 7 } $\begin{array}{c}\text { TX } \\
\text { Power } \\
{[\mathrm{dBm}]}\end{array}$ & $\begin{array}{c}\text { RX- } \\
\text { Power } \\
{[\mathrm{dBm}]}\end{array}$ & $\begin{array}{c}\text { Difference } \\
{[\mathrm{dB}]}\end{array}$ & $\begin{array}{c}\text { RX- } \\
\text { Power } \\
{[\mathrm{dBm}]}\end{array}$ & $\begin{array}{c}\text { Difference } \\
{[\mathrm{dB}]}\end{array}$ & $\begin{array}{c}\text { Difference } \\
{[\mathrm{dB}]}\end{array}$ \\
\hline \hline 3 & -40.8 & 3.8 & -44.1 & 7.1 & 2.4 \\
\hline 6 & -38.1 & 4.1 & -41.1 & 7.1 & 2.1 \\
\hline 10 & -35.5 & 5.5 & -37.2 & 7.2 & 0.8 \\
\hline 20 & -24.8 & 4.8 & -27.1 & 7.1 & 1.5 \\
\hline
\end{tabular}

\section{Antenna Measurement on Automat. Test Site}

Even though the antenna design methodology for $\mathrm{V} 2 \mathrm{~V}$ antennas is already well explored, predominantly the con- 


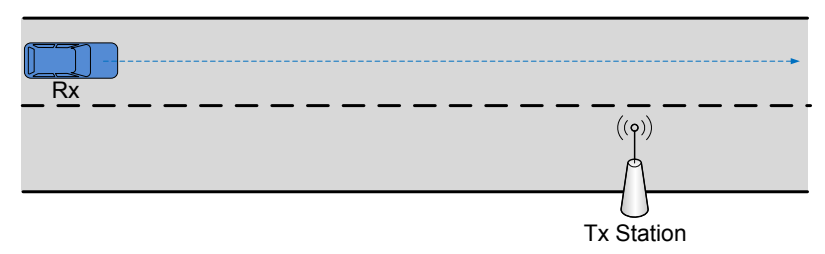

(a) Scenario 1: RX-Vehicle approaching Tx-Station in LOS

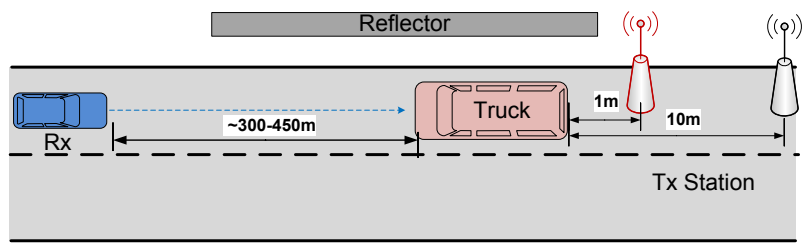

(b) Scenario 2 and 3: Rx-Vehicle and Tx-Station hidden by a truck at varying inter-distance $(1 \mathrm{~m}$ and $10 \mathrm{~m})$, side reflector only in scenario 3 .

Figure 3: Selected scenarios: Arrows indicate the direction of motion of the receiving vehicle.

ventional automotive mounting concepts impact the overall system performance metrics and significantly contribute to its limits [6, 10]. It is shown in [11] that proper antenna placement on the vehicle shall ultimately define the quality of the radio link and shall limit its performance metrics.

The desired omni-directional antenna coverage in azimuth conflicts with the conventional mounting positions on vehicles as depicted in Fig. 4. A relevant position for automotivecompliant $\mathrm{V} 2 \mathrm{~V}$ antenna is given by the conventional roof-top antenna module. This one is centered- and located at the back of the metallic vehicle roof. The vehicle roof itself provides some properties that negatively influence the radiation patterns of $\mathrm{V} 2 \mathrm{~V}$ antennas: It represents a metallic surface with finite dimensions and insets for non-metallic sun roofs or railings oriented in parallel to driving direction. In addition to the railings that cause shadowing to the left- and right sides of the vehicle, the roof provides a finite inclination $\vartheta_{\text {roof }}$ at the mounting position of the roof-top antenna. This angle depends on the vehicle type and can be in the range of $\vartheta_{\text {roof }} \in[10, \ldots, 15]^{\circ}$ for sedan cars as depicted in Fig. 4.

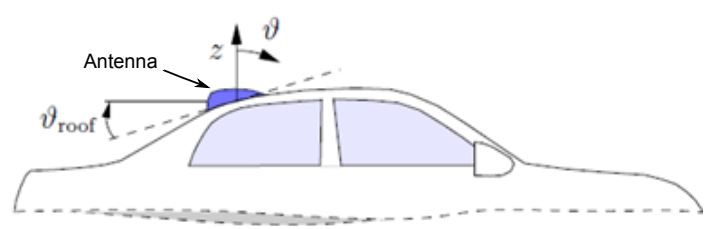

Figure 4: Mounting position of antenna module

As a result of the inclination, the overall embossing of the vehicle roof may therefore cause significant shadowing in driving direction. Therefore, the performance metrics of vehicular-mounted antennas in V2V communications depends on the type of the vehicle: Sedan- and convertible cars impose even more challenging requirements to the antenna design in comparison to truck- or minivan vehicles with an almost flat roof. Fig. 5 represents the results of the antenna radiation pattern for the automotive qualified roof-top antenna at $5.9 \mathrm{GHz}$. As can be seen from the antenna radiation performance, coverage towards driving direction in the range of $\varphi \in[330, \ldots, 30]^{\circ}$ is rather poor due to the presence of an integrated sun roof in the roof-top of the vehicle (light gray area on Fig. 5). This result is in accordance with [8].

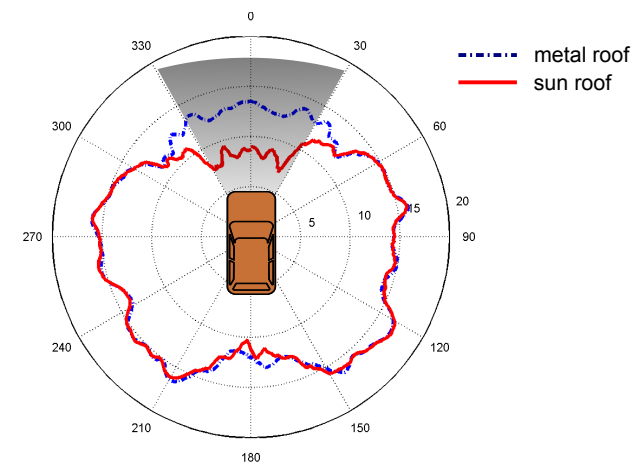

Figure 5: Plane radiation pattern of antenna module measured with a standard metallic roof (dash-dot line) and a sun roof (solid line) on RX-vehicle

\section{Field-Operational Test}

The results from the measurement campaign is presented in this section. All test scenarios have been conducted several times and we provide in this paper the average values. We evaluate the mean received power $P_{r}$ and the PDR against the distance $d_{T X-R X}$ between transmitter and receiver. The measured mean received power $P_{r}$ and the PDR for varying inter-distance $(1 \mathrm{~m}$ and $10 \mathrm{~m})$ between TX-Station and truck in a NLOS cases is shown in Fig. 6a, where a truck obstructed the LOS as described in scenario 2. We define a target PDR of at least $90 \%$ which should correspond to a fully reliable communication range. A variation of an interdistance from $1 \mathrm{~m}$ to $10 \mathrm{~m}$ between the shadowing truck and the TX-Station increases the effective communication range (ECR) from approximately $84 \mathrm{~m}$ to $168 \mathrm{~m}$ based on the definition of a reliable communication range (PDR $\geq 0.9$ ). Considering this fact, results confirm the assumption that interdistances have a significant impact on the maximum achievable communication range. Likewise, the effects of side reflectors, which substantially influence the results in this scenario, will be tested in scenario 3 .

Figure $6 \mathrm{~b}$ depicts the mean received power $P_{r}$ and the PDR with or without using side reflectors, considering an inter-distance between the TX-Station and truck of $1 \mathrm{~m}$ as described in scenario 3. As expected, side reflectors have a significant influence on the received power levels. Hence, we observe that the effective communication range is increased from approximatively $\mathrm{ECR} \approx 84 \mathrm{~m}$ (without side reflector) to 


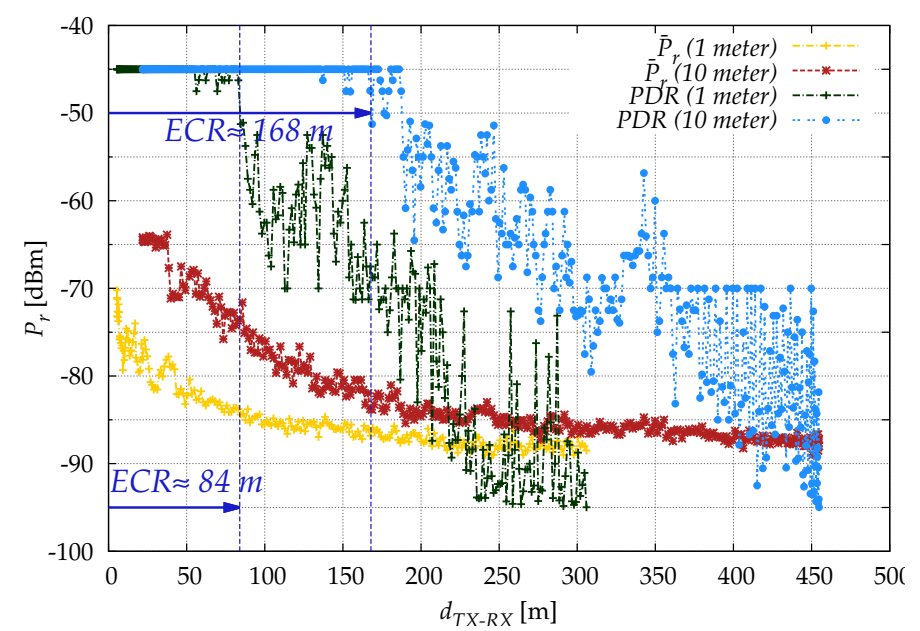

(a) Scenario 2: Impact of varying inter-distance (1 $\mathrm{m}$ and $10 \mathrm{~m})$, without side reflector.

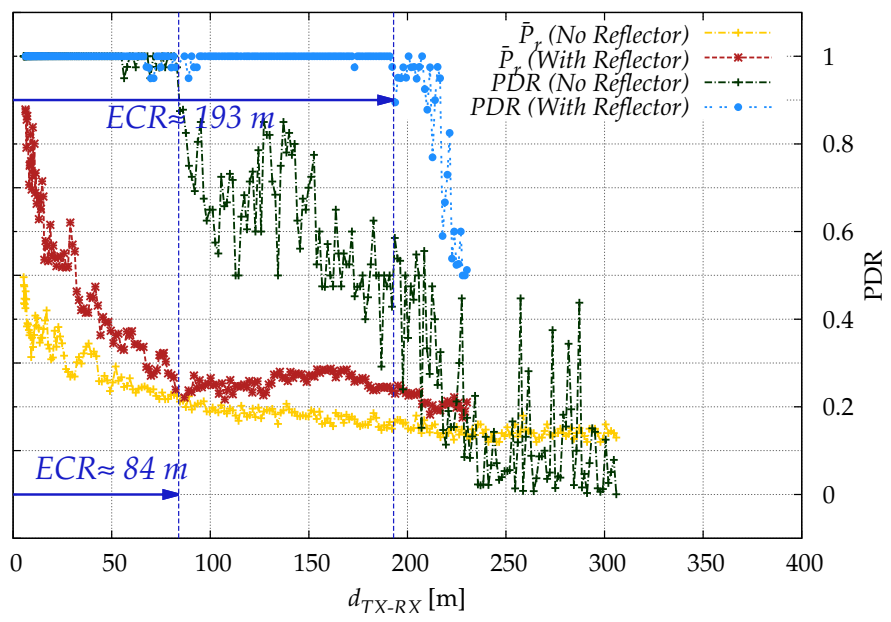

(b) Scenario 3: Impact of side reflector (inter-distance: $1 \mathrm{~m}$ )

Figure 6: Mean received power $\left(P_{r}\right)$ and mean PDR for scenario 2 and 3.

$\mathrm{ECR} \approx 193 \mathrm{~m}$ (with side reflector). However at higher distance $\left(d_{T X-R X}>230 m\right)$, this effect become less significant, indicating that the attenuation component caused by the decrease of the received signal power, which proportional to the inverse of the distance, has an amplitude that is probably quite high relative to reflected rays on the side reflector.

Figure 7 shows the mean received power $P_{r}$ as function of time obtained through experiments described in section III-B for scenario 1 . The maximum mean received power $P_{r}$ value, which is around $-51 \mathrm{~dB}$, correspond to the moment when the RX-vehicle has been directly in front of the TX-Station. An additional attenuation of the receiver signal power of about $5 \mathrm{~dB}$ can clearly be seen using a vehicle with a integrated sun roof. The difference is quite significant and constant from 0 up to 25 seconds but after that it becomes less significant, which is in accordance with the measured radiation pattern of the antenna as described in section $\mathrm{V}$. The results show that significant benefits can be achieved by differentiating between different types of vehicles according to their roof type.

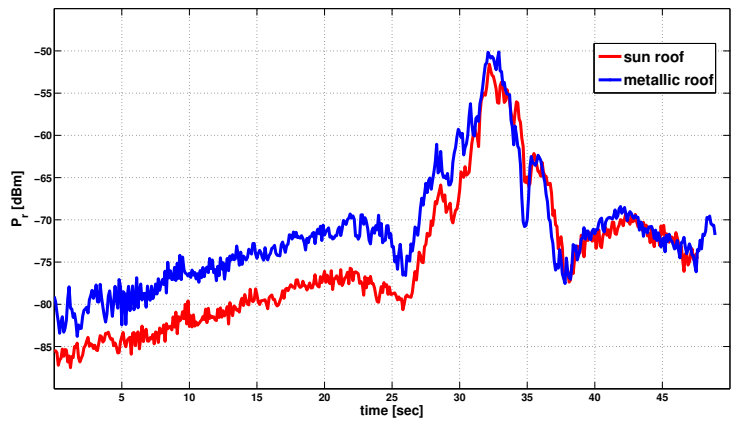

Figure 7: Mean received power $P_{r}$ with a standard metal roof (blue) and a sun roof (red) on RX-vehicle

\section{APPLICATION IMPACT}

We evaluate in this section the impact of the integration, antenna and channel effects on the link budget in a typical
ITS application. We selected the ITS RHW application, first from its safety-of-life aspect, but also as it will be the first ITS safety application to be deployed. The scenario description is depicted on Fig. 8.

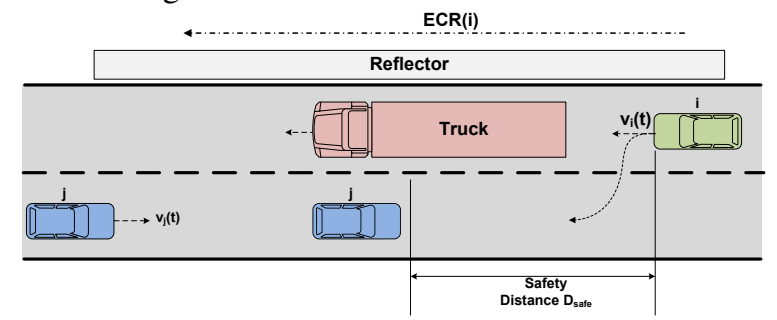

Figure 8: RHW application, considering vehicle $i$ hidden behind a truck to vehicle $j$ in an overtaking attempt

As we would like to test the influence of the integration and channel effects on the communication range, we consider one truck and one vehicle (green car on Fig. 8) driving on one direction, and another vehicle (blue) approaching on the opposite direction. Yet, the first vehicle is blocked visually and technologically by the truck immediately in front of it. The objective of the RHW application will be for the car hidden by the truck to detect the approaching vehicle and avoid overtaking it. From a communication point of view, such detection is defined as the reception of at least one beacon from the opposite vehicle before it reaches a safety critical distance beyond which an impact cannot be avoided, would the first car choose to overtake. We tested the performance of the RHW application first only considering the influence of the integrated link budget, and second by also including the beneficial aspect of side reflectors.

In combination with the ECR determined in section VI, we evaluated the empirical braking distance $D_{\text {safe }}$ and an additional Time-to-Collision $T T C_{a d d}$ as application-level metrics:

$$
D_{\text {safe }}[m]=\frac{(\text { speed } \cdot 3.6)^{2}}{100} \quad T T C_{a d d}[s]=\frac{\mathrm{ECR}-D_{\text {safe }}}{\text { speed }}
$$


The results depicted in Table IV should be read in two dimensions: first, the benefits from a longer inter-distance between vehicle $i$ and the truck. We see that by itself, it provides a higher opportunity for waves to avoid being blocked by the truck. In particular, at $1 \mathrm{~m}$ behind a truck, the RHW application fails, as ECR $<D_{\text {safe }}$. On the second dimension, we compare the benefit of the side reflectors. Here, we see that the metal side reflector provides waves with the opportunity to reach vehicle $j$ even at an inter-distance of $1 \mathrm{~m}$. In that case, we see that reflectors allow the application to succeed, as ECR $>D_{\text {safe }}$, providing even safer conditions than the $10 \mathrm{~m}$ without reflector $(\mathrm{ECR}=168 \mathrm{~m})$. From an ITS safety application perspective, it would be preferable to drive with at least $10 \mathrm{~m}$ leeway between vehicles, as it not only provides an increased safety distance, but it also significantly reduces the influence of mobile radio obstacle. Second, if the traffic conditions make drivers have a small inter-distance, then side reflectors are able to significantly help communicating 'around' any mobile road obstacle.

It can be noted that side reflectors are very frequent in vehicular environments. On highway, it can be the side noise attenuators, direction separators or even opposite traffic. In urban environment, side reflectors are building or parked cars on the side roads for instance.

Table IV: Impact of reflectors and inter-distance on RHW application (considering a vehicle driving at $100 \mathrm{~km} / \mathrm{h}$ and a dry asphalt for the breaking distance)

\begin{tabular}{l|c|c|c|c}
\hline Criteria & \multicolumn{2}{|c|}{ Without Reflector } & \multicolumn{2}{c}{ With Reflector } \\
\hline Inter-distance & $1 \mathrm{~m}$ & $10 \mathrm{~m}$ & $1 \mathrm{~m}$ & $10 \mathrm{~m}$ \\
\hline $\boldsymbol{D}_{\boldsymbol{s a f e}}[\boldsymbol{m}]$ & 100 & 100 & 100 & 100 \\
\hline $\boldsymbol{E C R}[\boldsymbol{m}]$ & 84 & 168 & 193 & N.A. \\
\hline $\boldsymbol{T T \boldsymbol { C } _ { \boldsymbol { a d d } \boldsymbol { d } } [ \boldsymbol { s } ]}$ & - & 2.4 & 3.3 & - \\
\hline
\end{tabular}

\section{CONCLUSION AND FUTURE WORK}

The objective of this paper was to investigate the joint impact of vehicular integration as well as wireless channel on the performance of DSRC in real experimental tests. We showed how vehicular integration effects, in particular cable, connector and chipset losses, as well as the influence of roof type, impact the communication link budget and have a significant influence on the communication range. We also showed that the type and distance between a mobile radio obstacle, as well as potential side-reflectors provide a beneficial influence on the communication range. From our study, leaving space between vehicles is not only safe, but provides also improved link reliability. Side reflectors also play a crucial beneficial role in improving the radio channel, and as such the link budget.

Considering the Road Hazard Warning (RHW) application, we showed that vehicular integration had a significant negative effect on its performance. Yet, we also illustrated that these effects could be compensated by beneficial channel effects such as side-road reflectors.
This work opens many doors, one in each link budget block, that we plan to explore in future work. Notably, we will further evaluate the impact of the antenna block, such as the antenna hood, or the influence of antenna positioning and type. We will also conduct a larger field test regarding the influence of the antenna angular radiation and channel reflectors on mobile and static radio obstacles. Our objective from these experiments is to extract models and parameters composing DSRC integrated in vehicles.

\section{REFERENCES}

[1] I. Sen and D. Matolak, "Vehicle-to-Vehicle Channel Models for the 5-GHz Band," IEEE Transactions on ITS, vol. 9, no. 2, pp. 235-245, 2008.

[2] A. Paier, J. Karedal, N. Czink, C. Dumard, T. Zemen, F. Tufvesson, A. Molisch, and C. Mecklenbräuker, "Characterization of Vehicle-to-Vehicle Radio Channels from Measurements at 5.2GHz," Wireless Personal Commun., vol. 50, no. 1, pp. 19-29, 2009.

[3] J. Kunisch and J. Pamp, "Wideband car-to-car radio channel measurements and model at $5.9 \mathrm{GHz}$," IEEE Vehicular Technol. Conf. (VTC 2008-Fall), Sept. 2008.

[4] J. Gozalvez, M. Sepulcre, and R. Bauza, "IEEE 802.11p Vehicle to Infrastructure Communications in Urban Environments," IEEE Comm. Mag.,, vol. 50, no. 5, pp. 176 -183 , may 2012.

[5] R. Meireles, M. Boban, P. Steenkiste, O. Tonguz, and J. Barros, "Experimental Study on the Impact of Vehicular Obstructions in VANETs," in IEEE Vehicular Networking Conference (VNC), 2010, pp. 338 -345.

[6] O. Klemp, "Performance Considerations for Automotive Antenna Equipment in Vehicle-to-Vehicle Communications," in URSI Int. Symp. Electromagnetic Theory, Berlin, Aug. 2010.

[7] M. Boban, R. Meireles, J. Barros, P. Steenkiste, and O. Tonguz, "Exploiting the Height of Vehicles in Vehicular Communication," in IEEE Vehicular Networking Conf., Amsterdam, Nov. 2011.

[8] A. Kwoczek, Z. Raida, J. Lacik, M. Pokorny, J. Puskely, and P. Vagner, "Influence of Car Panorama Glass Roofs on Car2Car Communication," IEEE Vehicular Networking Conference (VNC), 2011.

[9] "IEEE Standard for Information technology - Telecommunications and information exchange between systems - Local and metropolitan area networks - Specific requirements; Part 11: Wireless LAN Medium Access Control (MAC) and Physical Layer (PHY) Specifications," IEEE Std 802.11-2012, 2012.

[10] O. Klemp, A. Thiel, A. Paier, L. Bernado, J. Karedal, and A. Kwoczek, "In-Situ Vehicular Antenna Integration and Design Aspects for Vehicle-to-Vehicle Communications," in COST2100 Management Committee Meeting, Vienna, Austria, Sept. 2009.

[11] L. Reichardt, T. Fügen, and T. Zwick, "Influence of Antennas Placement on Car-to-Car Communications Channel," in EUCAP, 23-27 2009, pp. 630 -634. 